\title{
Tratamento de éguas receptoras de embriões visando sua utilização no segundo dia pós-ovulação ${ }^{1}$
}

\section{José Renato Costa Caiado², Francisco Aloízio Fonseca ${ }^{3}$, José Frederico Straggiotti Silva 4 , Reginaldo da Silva Fontes ${ }^{4}$}

1 Parte da tese de Doutorado do primeiro autor, apresentada à Universidade Estadual do Norte Fluminense (UENF), Campos dos GoytacazesRJ. Projeto financiado pela FENORTE.

2 Doutor em Zootecnia e Nutrição Animal da UENF.

3 Laboratório de Zootecnia e Nutrição Animal da UENF.

${ }^{4}$ Laboratório de Melhoramento Genético Animal da UENF.

Agradecimento: FENORTE e Bet Laboratories do Brasil.

RESUMO - Durante as estações de monta de 2001/2002 e 2002/2003, 43 éguas da raça Mangalarga Marchador receptoras de embriões foram tratadas aleatoriamente, via intramuscular, com $200 \mathrm{mg} / \mathrm{dia}$ de progesterona oleosa (P4) e 41 éguas da mesma raça receberam 0,044 mg/kg/dia de Altrenogest, aleatoriamente, via oral. Ambos os tratamentos foram efetuados entre D0 (dia da ovulação) e D5. As receptoras foram avaliadas no D2 e, se consideradas aptas, foram inovuladas. Dois grupos de 85 éguas da mesma raça foram utilizados aleatoriamente como controle e não receberam progestágeno, mas foram avaliados e inovulados em D2 ou D5, se considerados aptos. Os grupos D2 com P4 e D5 sem progestágeno tiveram maior número de éguas consideradas excelentes ou boas (aptas) no momento da avaliação, $55(64,71 \%)$ e $25(65,12 \%)$, respectivamente. O grupo D2 com Altrenogest apresentou número estatisticamente igual de receptoras aptas a inovulação e descartadas (20 e 21 éguas, respectivamente) no momento da avaliação. Nesta avaliação, no grupo D2 sem progestágeno, a quantidade de receptoras descartadas foi significativamente maior. A taxa de prenhez foi maior nos grupos D2 com P4 e D5 sem progestágeno (72,72 e 76,36\%, respectivamente). No grupo D2 com Altrenogest, obteve-se taxa de prenhez de 52,38\%, não-significativa, e, no grupo D2 sem progestágeno, a quantidade de éguas não-gestantes foi maior, embora não-significativa. O tratamento com P4 do D0 ao D5 possibilita antecipar a inovulação de receptoras para D2, com resultados semelhantes ao D5.

Palavras-chave: eqüino, Mangalarga Marchador, progestágenos, transferência de embriões

\section{Hormonal treatment of recipient mares of the Mangalarga Marchador breed for embryo transfer on the second day post ovulation}

\begin{abstract}
During the reproductive season of 2001 and 2002, 43 recipient mares of the Mangalarga Marchador breed were treated randomly with intra-muscular application of $200 \mathrm{mg} /$ day of oily progesterone (P4). Another group of $41 \mathrm{mares}$ of the same breed were treated randomly, via oral, with $0.044 \mathrm{mg} / \mathrm{kg} / \mathrm{day}$ of Altrenogest. Both treatments were performed between D0 (ovulation day) and D5. The recipients were evaluated on D2 and those in adequate conditions were in ovulated. Two groups of 85 mares of the same breed were used as control by not receive any progestogen. They were also evaluated and, when considered appropriate, in ovulated on D2 or D5. The groups D2/P4 and D5 without progestogen presented a significantly greater percentage of recipients considered appropriate during the evaluation, respectively $55(64.71 \%)$ and 25 (65.12\%). In the group D2 with Altrenogest, 20 mares were considered appropriate for in ovulation and 21 were not. The group D2 without progestogen showed a significantly higher percentage of mares considered not appropriate. The pregnancy rate after in ovulation for the groups D2/P4 and D5 without progestogen were respectively 72.72 and $76.36 \%$, significantly higher than those from the other treatments. The pregnancy rate for the group D2 with Altrenogest (52.38\%) was not significant. Despite a larger number of non pregnant recipients for the group D2 without progestogen, it was not significant The treatment of mares with P4 from D0 to D5 indicates it is possible to anticipate the in ovulation of recipient mares to D2 and to obtain similar results to when in ovulated on D5.
\end{abstract}

Key Words: equine, Mangalarga Marchador, embryo transfer, progestogen 


\section{Introdução}

Na espécie eqüina, a transferência de embriões tem sido a solução mais utilizada para aumento do número de descendentes/ano/matriz. No cenário mundial, o Brasil ocupa posição de destaque (3o lugar), acompanhando os dois líderes (Estados Unidos e Argentina) na utilização desta técnica (Squires et al., 2003).

A primeira transferência de embriões eqüinos (TEE) que logrou sucesso foi realizada no Japão por Oguri \& Tsutsumi (1972). No Brasil, os trabalhos foram iniciados em 1986, no estado de São Paulo, e foram realizados com sucesso na raça Mangalarga (Fleury, 1998b). Na raça Mangalarga Marchador, a técnica foi reconhecida e aceita pelo seu Conselho Deliberativo Técnico em 1995 e, desde então, tem sido muito utilizada (Caiado et al., 2005, 2000).

Hughes et al. (1980) estudaram o efeito da aplicação diária de progesterona oleosa via intramuscular em éguas ovariectomizadas e demonstraram que as doses de 50 e $100 \mathrm{mg} /$ dia não foram suficientes para atingir o nível plasmático considerado por Sharp (2000) satisfatório para manutenção da gestação (4 ng/mL). Esse nível foi consistentemente atingido com doses de $200 \mathrm{mg} / \mathrm{dia}$, confirmando que níveis séricos de progesterona inferiores a $1 \mathrm{ng} / \mathrm{mL}$ caracterizam o período do estro. Bergfelt \& Ginther (1996) verificaram em éguas e pôneis que a concentração de progesterona $(\mathrm{ng} / \mathrm{mL})$ foi próxima de zero no dia da ovulação e atingiu aproximadamente 3,0 no D2 e 11,0 no D7 em ambos os grupos de fêmeas. No D4, a concentração de progesterona foi menor nas éguas (em torno de 7,0 ng/mL) que nos pôneis (em torno de 9,0 ng/mL). Souza et al. (1999), trabalhando com potras da raça Brasileiro de Hipismo, encontraram concentrações médias de progesterona plasmática de 5,6 ng/mL no 40 dia pós-ovulação. Essas concentrações permaneceram elevadas até o 14으 dia, quando retornaram a valores basais.

Webel (1975) foi o primeiro pesquisador a reportar o uso de um progestágeno sintético via oral (Altrenogest) no controle do estro e da ovulação na égua. Desde então, têm-se observado numerosas citações sobre seu uso. Webel \& Squires (1982) administraram Altrenogest na dose de $0,044 \mathrm{mg} / \mathrm{kg}$ por 14 dias no período de transição do anestro invernal para a estação fisiológica de cobertura. Durante o tratamento, o estro foi suspenso em 262 de 278 éguas (94\%). Os autores concluíram que Altrenogest auxilia na normalização do ciclo estral no final da fase transicional. Outros pesquisadores descreveram o uso de progesterona injetável no controle do estro em éguas em fase de transição (Loy et al., 1981).
Pool et al. (1987) e Parry-Weeks \& Holtan (1987) utilizaram Altrenogest (progestágeno) visando à sincronização do estro em éguas receptoras de embrião. McKinnon et al. (1988) demonstraram que é possível a postergação da ovulação por meio da administração oral de Altrenogest. Almeida et al. (1995), em estudo com éguas no cio do potro, obtiveram bloqueio do cio e da ovulação em $100 \%$ das éguas e Squires et al. (1983) comprovaram que é possível suprimir o estro por tempo prolongado utilizando o mesmo progestágeno. Loy \& Swan (1966) testaram a aplicação de progesterona oleosa diariamente, via intramuscular, e relataram que, na dose de $100 \mathrm{mg}$ ou superior, o cio e a ovulação foram inibidos. Holtan et al. (1977), utilizando o mesmo produto, obtiveram resultados semelhantes.

A perda embrionária tem sido relacionada à baixa concentração de progesterona durante prenhez precoce (Douglas et al. 1985; Ginther, 1985). Valor inferior a 2,5 ng/mL no dia 12 foi usado como valor crítico para identificação de éguas com “disfunção luteal”. Hinrichs \& Kenney (1987) demonstraram que éguas ovariectomizadas tratadas com $300 \mathrm{mg} /$ dia de progesterona em óleo mantiveram a gestação após a transferência de embrião. ParryWeeks \& Holtan (1987) obtiveram o mesmo resultado usando Altrenogest na dose de $0,044 \mathrm{mg} / \mathrm{kg}$. Estes pesquis adores utilizaram os progestágenos por um período superior a 100 dias até que a placenta passou a suprir a necessidade fisiológica de progesterona necessária para manutenção da prenhez.

Aumentar o período útil de utilização de receptoras (atualmente é de 4 a 9 dias após-ovulação) é sempre um desafio que possibilita recompensas na redução de custos da técnica de TEE (Jasko, 2002). Entretanto, a utilização de receptoras em condições naturais antes de quatro dias após a ovulação não tem sido recomendada na literatura, observando-se que, a partir do $9 \underline{0}$ dia, a taxa de prenhez piora bastante após a transferência, inviabilizando a utilização a partir deste dia.

Es te trabalho foi realizado com os objetivos de estabelecer um protocolo de aplicação de progesterona (P4) ou Altrenogest por um curto período de tratamento, visando à utilização precoce de receptoras na transferência de embriões em eqüinos, e comparar os tratamentos com as duas drogas, indicando a mais eficiente.

\section{Material e Métodos}

O experimento foi desenvolvido em um criatório de eqüinos no município de Guarapari - ES, durante a estação de monta dos anos de 2001/2002 e 2002/2003. Como doadoras 
dos embriões, foram utilizadas 20 éguas Mangalarga Marchador com 3 a 18 anos de idade e condição corporal compatível com a atividade reprodutiva e, como receptoras, utilizaram-se 254 éguas da mesma raça, com idade de 3 a 12 anos e condição corporal compatível com a atividade reprodutiva. As éguas foram mantidas em pastagem de Brachiaria humidicula, com água e sal mineral à vontade, e receberam suplementação diária com capim-elefante (Penisetum purpureum) picado e concentrado (14\% de PB) em bretes com cochos individuais.

Uma vez ao dia, no mesmo brete de suplementação alimentar, as éguas foram rufiadas e submetidas a controle folicular e ultra-sonografia do trato reprodutivo (Scanner200 VetR - Pie Medical acoplado a um transdutor transretal linear de 7,5MHz).

O tônus uterino foi classificado de 1 a 4 , conforme os seguintes parâmetros:

tônus 1 - mínimo tônus do anestro até o início da atividade cíclica; o formato e a consistência do útero não estavam bem definidos ao exame de palpação retal;

tônus 2 - tônus proporcionado pela fase estrogênica do ciclo estral (estro); útero com consistência macia e formato quase tubular, com contratilidade ainda não perceptível ao toque;

tônus 3 - tônus da fase progesterônica do ciclo estral (diestro); útero com formato tubular bem definido, com aumento do tônus e da consistência e contratilidade uterina perceptível ao toque; e

tônus 4 - máximo tônus do início da prenhez, após o 13으 dia; contratilidade uterina mais perceptível ao toque e útero em formato tubular e consistência mais firme.

De acordo com a tonicidade, a cérvix foi classificada como aberta ou fechada. A abertura cervical foi constatada pela sua flacidez e mudança de formato ao toque, resultantes de baixa tonicidade, ocasionada pela ação de estrógenos. O fechamento, resultante da ação de progesterona no diestro e na gestação, ocasionando alta tonicidade (Hughes et al., 1977), foi observado pelo formato tubular e pela consistência firme ao exame.

As receptoras que ovularam e apresentaram persistência de folículos maiores que $20 \mathrm{~mm}$ ou ovários polifoliculares, tônus uterino de 1, 3 ou 4 e/ou fechamento cervical foram descartadas do experimento.

As doadoras foram inseminadas a partir do dia em que se detectou a presença de um folículo dominante com diâmetro igual ou maior que $35 \mathrm{~mm}$ até a ovulação (Caiado et al., 2005). A inseminação foi realizada em intervalos de 48 horas, na dose de 500×106 espermatozóides com motilidade progressiva, utilizando-se sêmen de um garanhão de conhecida fertilidade.
Os embriões foram coletados de forma não-cirúrgica entre os dias 6,5 e 8,5 após a ovulação da doadora (considerando-se D0 como o dia da ovulação), conforme procedimentos descritos por Squires et al. (2003), utilizando-se a solução de Ringer com lactato de sódio como meio de coleta (Alvarenga et al., 1993; Fleury, 1998a; Caiado et al., 2005).

Os embriões obtidos foram classificados em escala de 1 a 5, de acordo com McKinnon \& Squires (1988), em excelentes, bons, regulares, ruins e mortos ou ovócitos não-fecundados com auxílio de microscópio esterioscópico com aumento de 10 a 35 vezes.

No momento da transferência, as receptoras foram examinadas por meio de palpação retal e ultra-sonografia observando-se o tônus uterino e cervical, a morfologia dos corpos lúteos e a presença de edema, cistos, fluidos ou ar uterinos (Carnevale et al., 2000) - e classificadas como excelentes, boas, marginalmente aceitáveis ou inaceitáveis:

excelentes: pelo menos um corpo lúteo ecogenicamente bem definido, tônus uterino de 3 ou 4 , cérvix firmemente fechada e ausência de cistos, dobras e tumores uterinos;

boas: corpos lúteos nas condições supracitadas, com tônus uterino classificado como 3 ou 4, porém com dobra endometrial suave, pequenos cistos no útero ou cérvix menos firmemente fechada;

marginalmente aceitáveis: corpo lúteo presente, porém pequeno ou de imagem ecogenicamente ruim, útero com tônus entre 2 e 3 e cérvix com fechamento frouxo; e

inaceitáveis: receptoras sem corpo lúteo definido, com tônus uterino de 2 ou menos; presença de patologias uterinas e edema endometrial pronunciado, além de abertura cervical.

Somente as receptoras excelentes ou boas foram inovuladas neste experimento. A técnica utilizada foi a transcervical e não-cirúrgica (Riera et al., 1993, com modificações), com a deposição do embrião no corpo ou corno uterino, de acordo com a facilidade da inovulação. Para o diagnóstico de gestação, as receptoras foram examinadas por palpação retal e ultra-sonografia trans-retal aos 14 dias da ovulação de sua respectiva doadora e, quando o resultado foi positivo, o diagnóstico foi confirmado aos 21, 28 e 50 dias de gestação.

Após a ovulação, as receptoras foram divididas, aleatoriamente, em quatro tratamentos:

- D2 com P4: as receptoras (43 no total) foram tratadas com $200 \mathrm{mg}$ de progesterona em veículo oleoso (P4, Bet Laboratories do Brasil), via intramuscular, uma vez ao dia, do dia da ovulação (D0) até o D5, sendo avaliadas por meio de palpação retal e por exame ultra-sonográfico e inovuladas no D2; 
- D2 com Altrenogest: as receptoras (41) foram tratadas diariamente com $0,044 \mathrm{mg} / \mathrm{kg}$ de Altrenogest (Regumate ${ }^{\circledR}$ - Intervet), via oral, do D0 ao D5, sendo avaliadas por meio de palpação retal e exame ultra-sonográfico e inovuladas no D2;

- D2 sem progestágeno (controle D2): as receptoras (85) não receberam nenhum tratamento e foram avaliadas por meio de palpação retal e exame ultra-sonográfico e inovuladas no D2:

- D5 sem progestágeno (controle D5): as receptoras (85) também não receberam nenhum tratamento e foram avaliadas por meio de palpação retal e exame ultrasonográfico e inovuladas no D5.

Após a detecção da ovulação, iniciou-se, na tarde do mesmo dia, o tratamento com Altrenogest nas fêmeas dos grupos D2 com P4 e D2. As doses subseqüentes foram administradas nos outros dias pela manhã.

As coletas de sangue das receptoras para análise dos níveis de progesterona foram realizadas entre 10 e 12 horas, diariamente, do dia em que a ovulação foi detectada até o quinto dia após ovulação, continuando de 2 em 2 dias até o 130 dia. As amostras foram coletadas de cinco éguas, escolhidas ao acaso, em cada grupo experimental, por punção na veia jugular, em tubos de ensaio estéreis e heparinizados. Logo a seguir, foram centrifugadas a $1.500 \mathrm{~g}$ por 3 minutos para obtenção do plasma, que foi acondicionado em tubos de polipropileno e congelado a $-18^{\circ} \mathrm{C}$ até o dia da análise hormonal.

As concentrações séricas de progesterona foram obtidas por RIA (radioimunoensaio) utilizando-se kits comerciais (Coat-A-Count, DPC, Los Angeles, CA), conforme recomendações do fabricante, efetuando-se duplamente a análise de todas as amostras, que apresentaram coeficiente de variação menor que $5 \%$ dentro do ensaio. A dose mínima de progesterona detectável foi de $0,023 \mathrm{ng} / \mathrm{mL}$ de plasma.

Os resultados da classificação (excelente, boa, marginalmente aceitável ou inaceitável) e a taxa de prenhez obtidos nos quatro grupos de receptoras (D2 sem administração de progestágeno; D5 sem progestágeno; D2 com progesterona oleona; D2 com administração de Altrenogest) foram analisados conforme o teste qui-quadrado $(\mathrm{P}<0,05)$, considerando, como hipótese de nulidade, $50 \%$ de freqüência entre as classes. A hipótese alternativa pressupõe que o tratamento adotado altera a proporção igualitária entre as classes. Similarmente, analisou-se a taxa de prenhez.

Em cada tratamento, foram analisados, segundo o teste qui-quadrado $(\mathrm{P}<0,05)$, os números de receptoras com problemas no corpo lúteo, presença de folículos $>20 \mathrm{~mm}$, tônus uterino 1 e 2 , presença de dobras, edema e líquidos no útero e cérvix aberta. A hipótese de nulidade pressupõe $50 \%$ de freqüência entre as receptoras com e sem o problema identificado. A hipótese alternativa pressupõe que o tratamento adotado altera a proporção igualitária entre as classes.

As concentrações plasmáticas de progesterona foram analisadas em delineamento inteiramente casualizado com quatro tratamentos (D2 sem progestágeno, D5 sem progestágeno, D2 com progesterona oleosa e D2 com Altrenogest) e cinco repetições. Os resultados foram submetidos à análise de variância da regressão para os teores de progesterona no período de D0 a D13 do ciclo estral. Por meio de derivações, calculou-se o ponto de máximo das equações de regressão para cada repetição e os resultados foram submetidos à análise da variância e à comparação de médias pelo teste Tukey $(\mathrm{P}<0,05)$.

\section{Resultados e Discussão}

Na avaliação da genitália no tratamento D2 sem administração de progestágeno (controle D2), apenas $20(23,53 \%)$ das 85 éguas receptoras foram classificadas como excelentes ou boas para inovulação e, portanto, a maior parte foi descartada $(\mathrm{P}<0,05)$ por esse procedimento (Tabela 1$)$.

Contudo, na avaliação no D5, 55 (64,71\%) éguas foram classificadas como excelentes ou boas para inovulação, sendo que uma porção significativamente menor $(\mathrm{P}<0,05)$ foi descartada. Entre aquelas consideradas aptas para inovulação, nos grupos D2 e D5, respectivamente, 20 e 55 éguas foram utilizadas na transferência.

Os valores de progesterona endógena só atingiram os níveis de $4 \mathrm{ng} / \mathrm{mL}$, considerados suficientes para manutenção da prenhez (Sharp, 2000), a partir do D3, pois no D2, estes níveis se encontravam, em média, em $3 \mathrm{ng} / \mathrm{mL}$ (Bergfelt \& Ginther, 1996), justificando porque no D2 apenas pequena parte das éguas apresentou condições de ser inovulada, apesar da taxa de prenhez significativamente menor que naquelas inovuladas no D5.

Entre as 43 éguas tratadas com progesterona (P4), 28 $(65,12 \%)$ foram classificadas como excelentes ou boas no dia da inovulação $(\mathrm{P}<0,05)$. Depois da administração de Altrenogest, das 41 éguas tratadas apenas $21(51,22 \%)$ foram classificadas como excelentes ou boas no dia da inovulação $(\mathrm{P}>0,05)$. Foram inovuladas 22 éguas do grupo tratado com progesterona oleosa e 21 do grupo tratado com Altrenogest.

Neste experimento, a administração de progesterona (P4) em éguas receptoras aumentou $(\mathrm{P}<0,05)$ a proporção de receptoras classificadas como excelentes ou boas no D2, ampliando o período útil para inovulação destas receptoras. 
Tabela 1 - Classificação do sistema genital de éguas receptoras sob administração ou não de progestágeno do 1 으 (D0) ao 6 dia do ciclo estral (D5) e resultado da avaliação no 30 (D2) e no 60 dia do ciclo estral $(n=254)$

Table 1 - Classification of the genital system of recipient mares of the Mangalarga Marchador breed with or without administration of progestogen from the first (DO) to the sixth day of the estrous cycle (D5) and evaluation on the third and sixth days of the estrous cycle $(n=254)$

\begin{tabular}{|c|c|c|c|c|c|}
\hline \multirow[t]{2}{*}{$\begin{array}{l}\text { Dia da avaliação da } \\
\text { receptora sob } \\
\text { administração ou não } \\
\text { de progestágeno } \\
\text { Day of evaluation of recipient } \\
\text { with or without administration } \\
\text { of progestogen }\end{array}$} & \multirow[t]{2}{*}{$\begin{array}{l}\text { Número de éguas } \\
\text { Number of mares }\end{array}$} & \multicolumn{3}{|c|}{$\begin{array}{l}\text { Classificação do sistema } \\
\text { genital das receptoras no dia da inovulação } \\
\text { Número de casos (\%) } \\
\text { Classification of the genital system of } \\
\text { recipients on the day of in ovulation } \\
\text { Number of cases }(\%)\end{array}$} & \multirow[t]{2}{*}{$\begin{array}{l}\text { Número de } \\
\text { receptoras } \\
\text { inovuladas } \\
\text { Number of in ovulated } \\
\text { recipients }\end{array}$} \\
\hline & & $\begin{array}{c}\text { Marginalmente aceitável } \\
\text { ou inaceitável } \\
\text { Marginally acceptable } \\
\text { or rejected }\end{array}$ & $\begin{array}{l}\text { Excelente ou bom } \\
\text { Excellent or good }\end{array}$ & $\begin{array}{c}\text { Qui-quadrado } \\
\text { dentro de }(\mathrm{T}) \\
\text { Qui-square within }(T)\end{array}$ & \\
\hline D2 sem progestágeno & 85 & $65(76,47 \%)$ & $20(23,53 \%)$ & $23,82 *$ & 20 \\
\hline $\begin{array}{l}\text { D5 sem progestágeno } \\
\text { D5 som }\end{array}$ & $30(35,29 \%)$ & $55(64,71 \%)$ & $7,35^{*}$ & 55 & \\
\hline $\begin{array}{l}\text { D5 without progestogen } \\
\text { D } 2 \text { com } \mathrm{P} 4\end{array}$ & $\begin{array}{c}85 \\
15(34,88 \%)\end{array}$ & $28(65,12 \%)$ & $3,93^{*}$ & 22 & \\
\hline D2 with P4 & 43 & & & & \\
\hline $\begin{array}{l}\text { D2 com Altrenogest } \\
\text { D2 with Altrenogest }\end{array}$ & 41 & $20(48,78 \%)$ & $21(51,22 \%)$ & $0,02^{\mathrm{ns}}$ & 21 \\
\hline Total & 254 & 130 & 124 & - & 118 \\
\hline
\end{tabular}

Desse modo, as receptoras submetidas a esse tratamento apresentaram desempenho similar ao daquelas avaliadas no D5, controle utilizado neste experimento, que está dentro do período utilizado pela maioria dos pesquisadores em transferência de embriões em eqüinos, como Schmidtet al. (1995), Squires et al. (1998), McKinnon (1999), Squires et al. (2003) e Jasko (2002).

Para avaliação dos principais problemas no sistema genital das éguas classificadas como marginalmente aceitáveis ou rejeitadas, no dia da avaliação das receptoras tratadas ou não com progestágeno, foram calculadas as porcentagens de ocorrência do problema em relação ao número de éguas utilizadas no grupo (Figura 1). Entre as receptoras não tratadas com progestágeno, $25(29,4 \%)$ apresentaram problemas no corpo lúteo no D2 e $15(22,2 \%)$ no D5. Não houve diferença $(\mathrm{P}>0,05)$ entre os diferentes grupos tratados com progestágeno; foram observados problemas no corpo lúteo de $14(32,6 \%)$ éguas tratadas com P4 e de 10(24,4\%) tratadas com Altrenogest. Praticamente $100 \%$ das estruturas que originam os corpos lúteos são visíveis no dia da ovulação e assim permanecem até o D10, quando começam a diminuir em proporção. A metade dos corpos lúteos se forma com uma massa não-ecogênica em seu interior (sangue e fibrina), a qual diminui progressivamente à medida que o tecido ecogênico se forma (Ginther, 1986), justificando porque na avaliação no D5 foi encontrada a menor porcentagem de problemas, embora não-significativa.

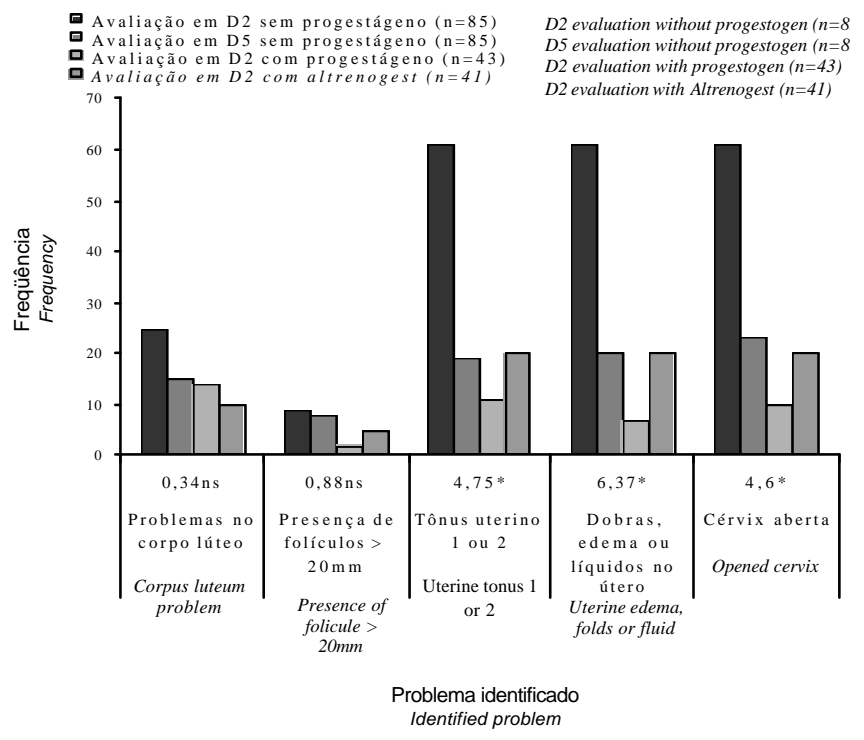

Figura 1 - Porcentagem de características indesejáveis no sistema genital de éguas receptoras (254) de embriões tratadas ou não com progestágeno e avaliadas no dia D2 e no D5 pós-ovulação. * Teste qui-quadrado entre problemas identificados, significativo $(G L=1, P<0,05)$. ns - não-signifcativo.

Figure 1 - Percentage of undesirable characteristics of the genital system of recipient mares of the Mangalarga Marchador breed evaluated on the days D2 and D5, with or without administration of progestogen ( $n=254$ mares). * Qui-square test between identified problems, significant $(G L=1, P<0,05)$. ns - notsignificant. 
As proporções de folículos com diâmetro superior a $20 \mathrm{~mm}$ nos tratamentos sem progestágeno foram de $9(15,5 \%)$ para D2 e $8(11,1 \%)$ para D5, enquanto, no grupo tratado com P4 foi de $2(4,7 \%)$ e naquele tratado com Altrenogest foi de $5(12,2 \%)$ no D2, o que indica baixa ocorrência e nenhuma diferença entre os grupos $(\mathrm{P}>0,05 \%)$. Os folículos com diâmetro entre 2 e $5 \mathrm{~mm}$ aparecem um pouco antes da ovulação, ao passo que os maiores, de diâmetro superior a $16 \mathrm{~mm}$, surgem no meio do diestro e sofrem atresia ou ovulam (Ginther, 1986; Gastal et al., 1997), o que explica sua baixa ocorrência no início do diestro neste experimento. A maior porcentagem do problema foi observada no grupo D2 sem progestágeno e a menor, no grupo D2 com $\mathrm{P} 4$, provavelmente em virtude das baixas concentrações de progesterona endógena neste dia (Bergfelt \& Ginther, 1996), hormônio inibitório para o crescimento folicular.

Entre as receptoras que não receberam progestágeno, $61(71,8 \%)$ apresentaram baixo tônus uterino no D2 e 19 $(28,1 \%)$ no D5. Daquelas tratadas com progestágeno e avaliadas no D2, $11(25,8 \%)$ e $20(48,8 \%)$ tratadas com P4 e Altrenogest, respectivamente, apresentaram baixo tônus uterino $(\mathrm{P}<0,05)$.

Nos tratamentos sem administração de progestágeno, foram observados dobras e edemas do endométrio em 61 éguas $(71,8 \%)$ avaliadas no D2 e em $20(28,9 \%)$ avaliadas no D5. Do mesmo modo, sete receptoras tratadas com P4 $(16,3 \%)$ e 20 tratadas com Altrenogest $(48,8 \%)$ apresentaram esses problemas ao D2. Nos tratamentos sem administração de progestágeno, observou-se ainda abertura cervical em $61(71,8 \%)$ receptoras no D2 e em $23(32,6 \%)$ avaliadas no D5. Esse comportamento, nos grupos sob administração de progestágeno, foi verificado em apenas $10(23,3 \%)$ receptoras tratadas com $\mathrm{P} 4$ e em $20(48,8 \%)$ que receberam Altrenogest.

A porcentagem de receptoras dos grupos D2 com P4 e D5 sem progestágeno que apresentaram tônus uterino insuficiente, cérvix aberta e edema, dobras e líquidos no útero foi menor que nos outros grupos $(\mathrm{P}<0,05)$, o que comprova a eficiência do hormônio natural, endógeno ou exógeno, no estabelecimento do tônus característico desta fase do ciclo estral. Estes resultados reforçam a afirmação de Bonafos et al. (1994) de que o progesterona é o único hormônio produzido pelo corpo lúteo necessário para o desenvolvimento da turgidez uterina na égua. Pelehach et al. (2002) também demonstraram a importância do progesterona na dissipação do edema uterino. Além disso, McCue et al. (1999) e Carnevale et al. (2000) concluíram que as receptoras selecionadas para inovulação, após o exame de palpação retal e ultra-sonografia, apresentaram concentrações de progesterona significativamente mais altas em comparação àquelas marginalmente aceitáveis ou rejeitadas . Segundo esses autores, os baixos níveis de progesterona plasmáticos estão relacionados ao inadequado tônus uterino e/ou cervical, podendo constituir um parâmetro para descarte de receptoras para transferência de embriões.

Vários autores têm demonstrado a eficácia do Altrenogest como progestágeno sintético (Webel, 1975; Webel \& Squires, 1982; Pool et al., 1987; Parry-Weeks \&

Tabela 2 - Porcentagem de prenhez de éguas receptoras tratadas ou não com progestágeno do 1으 (D0) ao 6음 dia do ciclo estral (D5) e resultados da avaliação no 30 (D2) e no 60 dia do ciclo estral

Table 2 - Pregnancy rate of recipient mares of the Mangalarga Marchador breed with or without administration of progestogen from first (D0) to sixth day of estrous cycle (D5) and evaluation on the third (D2) and sixth days of the estrous cycle

\begin{tabular}{|c|c|c|c|c|c|}
\hline \multirow{2}{*}{$\begin{array}{l}\text { Dia da avaliação da receptora tratada } \\
\text { ou não com progestágeno } \\
\text { Day of evaluation of recipient with } \\
\text { or without progestogen administration }\end{array}$} & \multicolumn{3}{|c|}{$\begin{array}{l}\text { Número de inovulações e prenhez } \\
\text { Number of in ovulations and pregnancy }\end{array}$} & \multirow[t]{2}{*}{$\begin{array}{c}\text { Taxa de prenhez }(\%) \\
\text { Pregnancyrate }(\%)\end{array}$} & \multirow[t]{2}{*}{$\begin{array}{c}\text { Valor qui-quadrado }\left(\mathrm{c}^{2}\right) \\
\text { Qui-square value }\left(c^{2}\right)\end{array}$} \\
\hline & $\begin{array}{l}\text { Inovuladas } \\
\text { Inovulated }\end{array}$ & $\begin{array}{c}\text { Gestantes } \\
\text { Pregnant }\end{array}$ & $\begin{array}{l}\text { Não-gestantes } \\
\text { Not pregnant }\end{array}$ & & \\
\hline D2 sem progestágeno & 20 & 6 & 14 & 30,00 & $3,2^{\mathrm{ns}}$ \\
\hline $\begin{array}{l}\text { D2 with progestogen } \\
\text { D5 sem progestágeno } \\
\text { D5 with progestogen }\end{array}$ & 55 & 42 & 13 & 76,36 & $15,29 *$ \\
\hline $\begin{array}{l}\mathrm{D} 2 \text { com } \mathrm{P} 4 \\
\text { D2 with } P 4\end{array}$ & $\begin{array}{l}16 \\
22\end{array}$ & 6 & 72,72 & $4,55^{*}$ & \\
\hline D2 com Altrenogest & 21 & 11 & 10 & 52,38 & $0,05^{\mathrm{ns}}$ \\
\hline $\begin{array}{l}\text { D2 with Altrenogest } \\
\text { Total } \\
\text { Teste qui-quadrado para (T): } 11,54 *\end{array}$ & 118 & 75 & 43 & 63,53 & - \\
\hline
\end{tabular}

* Teste qui-quadrado entre tipos de problemas, significativo $(G L=1, P<0,05)$. ns- não-signifcativo.

${ }^{*}$ Qui-square test between types of problems, significant ( $\left.G L=1, P<0.05\right)$. ns- not-significant 
Tabela 3 - Média e desvio-padrão das concentrações plasmáticas de progesterona ( $\mathrm{ng} / \mathrm{mL})$, de acordo com o dia do ciclo estral, em receptoras Mangalarga Marchador

Table 3 - Mean \pm standard deviation of plasma progesterone concentration $(\mathrm{ng} / \mathrm{mL})$ in relation to the day of the estrous cycle of recipient mares of the Mangalarga Marchador breed

\begin{tabular}{|c|c|c|c|c|}
\hline \multirow[b]{2}{*}{$\begin{array}{l}\text { Dia do ciclo estral } \\
\text { Day of the estrous cycle }\end{array}$} & \multicolumn{4}{|c|}{$\begin{array}{c}\text { Tratamento* } \\
\text { Treatment }\end{array}$} \\
\hline & $\begin{array}{l}\text { D2 sem progestágeno } \\
\text { D2 with progestogen }\end{array}$ & $\begin{array}{l}\text { D5 sem progestágeno } \\
\text { D5 with progestogen }\end{array}$ & $\begin{array}{l}\mathrm{D} 2 \text { com } \mathrm{P} 4 \\
\text { D2 with } P 4\end{array}$ & $\begin{array}{l}\text { D2 com Altrenogest } \\
\text { D2 with Altrenogest }\end{array}$ \\
\hline D0 & $2,51 \pm 2,56^{\mathrm{a}}$ & $2,06 \pm 2,15^{\mathrm{a}}$ & $1,84 \pm 3,02^{\mathrm{a}}$ & $0,46 \pm 0,35^{\mathrm{a}}$ \\
\hline D1 & $3,41 \pm 2,77^{b}$ & $4,25 \pm 2,64^{b}$ & $17,60 \pm 14,13^{\mathrm{a}}$ & $2,12 \pm 0,87^{\mathrm{b}}$ \\
\hline D2 & $5,61 \pm 1,41^{\mathrm{a}}$ & $6,40 \pm 1,53^{\mathrm{a}}$ & $8,71 \pm 1,60^{\mathrm{a}}$ & $5,35 \pm 2,60^{\mathrm{a}}$ \\
\hline D5 & $9,86 \pm 4,56^{\mathrm{a}}$ & $10,65 \pm 4,32^{\mathrm{a}}$ & $13,02 \pm 3,67^{\mathrm{a}}$ & $6,35 \pm 4,19^{a}$ \\
\hline D7 & $11,85 \pm 4,09^{\mathrm{a}}$ & $11,88 \pm 3,23^{\mathrm{a}}$ & $12,10 \pm 2,66^{\mathrm{a}}$ & $10,61 \pm 3,48^{a}$ \\
\hline D9 & $8,37 \pm 0,60^{\mathrm{a}}$ & $9,38 \pm 1,66^{\mathrm{a}}$ & $10,76 \pm 1,84^{\mathrm{a}}$ & $8,81 \pm 0,57^{\mathrm{a}}$ \\
\hline D 11 & $9,29 \pm 2,01^{\mathrm{a}}$ & $11,48 \pm 2,33^{\mathrm{a}}$ & $8,71 \pm 0,84^{a}$ & $7,51 \pm 3,87^{\mathrm{a}}$ \\
\hline D 13 & $8,09 \pm 3,03^{\mathrm{a}}$ & $7,30 \pm 5,26^{\mathrm{a}}$ & $8,10 \pm 1,70^{\mathrm{a}}$ & $6,32 \pm 4,41^{\mathrm{a}}$ \\
\hline
\end{tabular}

* Médias seguidas de mesma letra na linha não diferem $(P<0,05)$ pelo teste Tukey.

* Means followed of the same letters in the same row do not differ $(P<0.05)$ by the Tukey test.

Holtan, 1987; McKinnon et al., 1988; Almeida et al., 1995), contudo, neste experimento, seu efeito não foi estatisticamente comprovado $(\mathrm{P}>0,05)$, embora tenha se distinguido em termos numéricos no grupo D2 sem progestágeno.

Na Tabela 2 constam os dados referentes à taxa de prenhez de éguas receptoras sob a administração ou não de progestágeno. As maiores $(\mathrm{P}<0,05)$ taxas de prenhez foram observadas nas éguas não tratadas com progestágeno e inovuladas no D5 $(76,36 \%)$ e naquelas tratadas com P4 e inovuladas no D2 (72,72\%).

As taxas obtidas corroboram as encontradas em trabalhos recentes da literatura para transferência não-cirúrgica de embriões eqüinos a fresco: Squires et al. (1998) obtiveram 69 a 89\%; Jasko (2002), 62,5 a 100\%; Carney et al. (1991), 60 a 71\%; e Carnevale et al. (2000), 65,7\% de prenhez. Apesar dos valores não-significativos $(\mathrm{P}>0,05)$, entre as éguas inovuladas no D2, verificou-se $52,38 \%$ de prenhez naquelas tratadas com Altrenogest e 30\% naquelas não tratadas com progestágeno.

As receptoras do grupo $\mathrm{D} 2$ com $\mathrm{P} 4$ apresentaram pico de progesterona plasmática mais elevado, além de precocidade em relação aos demais grupos (Tabela 3 ).

Bergfelt \& Ginther (1996) afirmaram que a concentração de progesterona $(\mathrm{ng} / \mathrm{mL})$ em éguas e pôneis foi próxima de zero no dia da ovulação e atingiu aproximadamente 3,0 noD2 e 11,0 no D7. A concentração de progesterona plasmática nesse experimento alcançou, no D2, médias de 5,61士1,41; $6,40 \pm 1,53$ e 5,35 $\pm 2,60$, respectivamente, nos grupos D2 sem progestágeno, D5 sem progestágeno e D2 com Altrenogest. Esses resultados são superiores aos descritos por Bergfelt \& Ginther (1996), todavia, neste experimento, as amostras de sangue foram coletadas apenas uma vez ao dia, mas existem
3,33 pulsos diários de liberação de progesterona (Perkins et al., 1993). Além disso, as coletas deste experimento foram realizadas sempre das 10 às $12 \mathrm{~h}$ (a primeira no dia em que a ovulação foi detectada) por meio de toque retal uma vez ao dia; com esse procedimento, a coleta em algumas éguas pode ter sido realizada a mais de 20 horas pós-ovulação, o que, associado às variações raciais, pode justificar as altas taxas de progesterona em algumas das éguas na primeira coleta e aumento dos valores médios esperados. Os valores mais elevados de progesterona em D2 encontrados nesses grupos não qualificam estas receptoras para inovulação no mesmo dia, pois, de acordo com Bergfelt \& Ginther (1996), a concentração considerada suficiente para manter a prenhez, de 4 ng/mL (Sharp, 2000), atingida no D3 não resultou em recomendações para inovulação de receptoras nesse mesmo dia. A literatura consultada recomenda a inovulação a partir do D4 (Caiado et al., 2005; Carnevale et al., 2000; Jasko, 2000; Squires et al., 2003).

A concentração média de progesterona no D1 no grupo D2 com P4 $(17,60 \pm 14,13 \mathrm{ng} / \mathrm{mL})$ foi maior $(\mathrm{P}<0,05)$ que nos demais grupos, o que pode ser justificado pelo fato de que, além do progesterona endógeno, foi administrado progesterona natural (P4). Segundo Hughes et al. (1980), níveis plasmáticos médios de progesterona superiores a $4 \mathrm{ng} / \mathrm{mL}$ podem ser alcançados em éguas ovariectomizadas submetidas à aplicação diária de 200 mg de progesterona oleosa. O grupo estudado recebeu dose de $200 \mathrm{mg} / \mathrm{dia}$ de progesterona oleosa, além da naturalmente produzida.

O Altrenogest, por sua vez, é detectado na análise hormonal pelo método utilizado neste experimento, pois não apresenta similaridade com a molécula de progesterona. Assim, a concentração de progesterona nesse grupo de 
receptoras manteve-se de acordo com a dos grupos sem administração de progestágeno.

As análises da variância para o ponto máximo das equações de regressão indicaram a existência de efeitos significativos $(\mathrm{P}<0,05)$ entre os grupos de receptoras quanto ao dia do ciclo estral em que ocorre o pico de concentração plasmática de progesterona. Todavia, não existem efeitos $(\mathrm{P}>0,05)$ entre os grupos quanto à concentração máxima alcançada.

\section{Conclusões}

O tratamento com progesterona $(\mathrm{P} 4)$ em receptoras eqüinas no período de D0 (dia da ovulação) a D5 possibilitou a inovulação destas receptoras no D2 e a obtenção de taxa de prenhez estatisticamente similar à de éguas consideradas excelentes e boas para inovulação no D5.

O tratamento com Altrenogest no mesmo período, em comparação ao grupo D2 sem progestágeno, aumentou a taxa de prenhez, contudo, esse aumento não foi estatisticamente significativo.

O protocolo proposto neste experimento mostrou-se eficiente para aplicação de $\mathrm{P} 4$, em receptoras eqüinas do D0 a D5 e inovulação no D2, com taxas similares às do grupo controle.

\section{Literatura Citada}

ALMEIDA, F.Q.; FONSECA, F.A.; ESPECHIT, C.J.B. Efeitos da prostaglandina F-2 alfa e da progesterona na eficiência reprodutiva de éguas mestiças no período pós parto. Revista da Sociedade Brasileira de Zootecnia, v.24, n.4, p.652659,1995

ALVARENGA, M.A.; LANDIN e ALVARENGA, F.C.; MEIRA, C. Modification in the technique used to recovery equine embryos. Equine Veterinary Journal, v.15, p.111-112, 1993.

BERGFELT, D.R.; GINTER, O.J. Ovarian, uterine and embryo dynamics in horses versus ponies. Journal of Equine Veterinary Science, v.16, n.2, p.66-72, 1996

BONAFOS, L.D.; CARNEVALE, E.M.; SMITH, C.A. et al. Development of uterine tone in nonbred and pregnant mares. Theriogenology, v.42, p.1247-1255, 1994

CAIADO, J.R.C.; FONSECA, F.A.; SILVA, J.F.S. et al. Aplicação do flunixin meglumine antes da transferência não-cirúrgica de embriões em éguas da raça Mangalarga Marchador. Revista Brasileira de Ciências Veterinárias, v.12, n.13, p.11-15, 2005.

CARNEY, N.J.; SQUIRES, E.L.; COOK, V.M. et al. Comparison of pregnancy rates from transfer of fresh versus cooled, transported equine embryos. Theriogenology, v.36, p.23-31, 1991.

CARNEVALE, E.M.; RAMIRES, R.J.; SQUIRES, E.L. et al. Factors affecting pregnancy rates and early embryonic death after equine embryo transfer. Theriogenology, v.54, p.965-979, 2000.

DOUGLAS, R.H.; BURNS, P.J.; HERSHMAN, L. Physiological and commercial parameters for producing progeny for subfertile mares by embryo transfer. Equine Veterinary Journal, v.3, p.111-114, 1985.

LEURY, J.J. O dia da colheita na taxa de recuperação embrionária em eqüinos em uma central de transferência de embriões comercial. Arquivo da Faculdade de Veterinária da UFRGS v.26, n.1, p.268, 1998a.

FLEURY, J.J. Transporte de embriões eqüinos e sua aplicação no Brasil. Arquivo da Faculdade de Veterinária da UFRGS v.26, n.1, p.264, 1998b.

GASTAL, E.I.; GASTAL, M.O.; NOGUEIRA, G.P. et al. Role of diameter differences among follicles in selection of a future dominant follicle in mares. Biology of Reproduction, v. 57 p.1320-1372, 1997.

GINTHER, O.J. Ultrassonic imaging and reproductive events in the mares. 3.ed. Madison: Equiservice, 1986. 378p.

GINTHER, O. Embryonic loss in mares: Incidence, time of occurrence and hormonal involvement. Theriogenology, v.23, p.77-83, 1985.

HINRICHS, K.; KENNEY, M. Effect of timing of progesterone administration on pregnancy rate after embryo transfer in ovariectomized mares. Journal of Reproduction and Fertility, v.35, p.439-443, 1987.

HOLTAN, D.W.; DOUGLAS, R.H.; GINTHER, O.J. Estrus, ovulation and synchronization whith progesterone, prostaglandin F2 alfa and human chorionic gonadotrofin in pony mares. Journal of Animal Science, v.44, n.3, p.431-437, 1977.

HUGHES, J.P.; STABENFELT, G.H.; KENNEDY, P.C. The estrous cycle and selected functional and pathologic ovarian abnormalities in the mare. The Veterinary clinics of North America (Large Animal Practice), v.2, n.2, p.225-239, 1980.

HUGHES, J.P.; STABENFELDT, G.H.; EVANS, J.W. The oestrus cycle in the mare and its uterine control. Australian Veterinary Journal, v.53, p.415-419, 1977.

JASKO, D.J. Comparison of pregnancy rates following nonsurgical transfer of day 8 equine embryos using various transfer devices. Theriogenology, v.58, p.713-715, 2002.

LOY, R.G.; PEMSTEIN, R.; O'CANNA, D. et al. Control of ovulation in cycling mares with ovarian steroids and prostaglandin. Theriogenology, v.15, p.191-200, 1981.

LOY, R.G.; SWANN, S.M. Effects of exogenous progestogens on reproductive phenomena in mares. Journal of Animal Science, v.25, p.821-826, 1966.

MCCUE. P.M.; VANDERWALL, D.K.; KEITH, S.L. et al. Equine embryo transfer: influence of endogenous progesterone concentration in recipient on pregnancy outcome. Theriogenology, v.51, p.265-275, 1999

MCKINNON, A.O. Breeding and its technology - now and the future [1999]. World Trotting Conf. Papers. Disponível em <http://www.harness.org.au/99wldcon/PMCKINN5.HTM.> Acesso em: 15/12/2004.

MCKINNON, A.O.; SQUIRES, E.L.; CARNEVALE, E.M. et al. Ovariectomized steroid-treated mares as embryo transfer recipients and as model to study the role of progestin in pregnancy maintenance. Theriogenology, v.29, p.1011-1063, 1988.

MCKINNON, A.O.; SQUIRES, E.L. Morfologic assesment of the equine embryo. Journal of American Veterinary Medical Association, v.192, n.3, p.401-406, 1988.

OGURI, N.; TSUTSUMI, Y. Nonsurgical recovery of equine eggs, and an attempt at nonsurgical egg transfer in horses. Journal of Reproduction and Fertility, v.31, p.187-195, 1972.

PARRY-WEEKS, L.C.; HOLTAN, D.W. Effect of Altrenogest on pregnancy maintenance in unsynchronized equine embryo recipient. Journal of Reproduction and Fertility, v.35, p.443-448, 1987.

PELEHACH, L.M.; GREAVES, H.E.; PORTER, M.B. et al. The role of estrogen and progesterone in the induction and dissipation of uterine edema in mares. Theriogenology, v.58, p.441-444, 2002. 
PERKINS, N.R.; THRELFALL, W.R.; OTTOBRE, J. S. Pulsatile secretion of luteinizing hormone and progesterone in mares during the estrous cycle and early pregnancy. American Journal of Veterinary Research, v.54, n.11, p.1929-1934, 1993.

POOL, K.F.; WILSON, J.M.; WEEB, G.W. et al. Exogenous hormone regimens to utilize sucessfully mares in diestrus (days 2-14 after ovulation) as embryo transfer recipients. Journal of Reproduction and Fertility, v.35, p.429-432, 1987.

RIERA, F.L.; McDONOUGH, J. Commercial embryo transfer in polo ponies in Argentina. Equine Veterinary Journal, v.15, p.116-1118, 1993.

SCHIMIDT, A.R.; THAYER, J.; CARLETON, C.L. Stored, cooled embryos: a successful alternative to immediate embryo transfer. Equine Pratice, v.4, p.381-386, 1995.

SOUZA, J.A.T.; GACEK, F.; OLIVEIRA, J.B. Características do corpo lúteo e progesterona plasmática no ciclo estral de potras da raça Brasileiro de Hipismo. Arquivo da Faculdade de Veterinária da UFRGS, v.27, p.298, 1999.

SHARP, D.C. The early fetal life of the equine conceptus. Animal Reproduction Science, v.60-61, p.679-689, 2000.
SQUIRES, E.L.; CARNEVALE, E.M.; MCCUE, P.M. et al. Embryo technologies in the horse. Theriogenology, v.59, p.151-170, 2003.

SQUIRES, E.L.; SHIDELER, R.K.; VOSS, J.L. et al. Clinical applications of progestin in mares. Compendium on Continuing Education for the Veterinary Practitioners, v.5, p.516-522, 1983.

SQUIRES, E.L.; MCCUE, P.M.; VANDERWALL, D. The current of status of equine embryo transfer. Theriogenology, v.51, p.91-104, 1998.

WEBEL, S.K. Estrous control in horses with a progestin.Journal of Animal Science, v.41, p.385, 1975.

WEBEL, S.K.; SQUIRES, E.L. Control of the oestrous cycle in mares with Altrenogest. Journal of Reproduction and Fertility, v.32, p.193-198, 1982. 